\title{
Téoros
}

Revue de recherche en tourisme

\section{Habitudes de voyage des Québécois et des Ontariens dans les pays où l'hépatite $A$ est endémique}

\section{Gaston De Serres, Bernard Duval, Ramak Shadmani et Isabelle Rouleau}

Volume 24, numéro 3, automne 2005

Tourisme et santé, quelques perspectives

URI : https://id.erudit.org/iderudit/1071075ar

DOI : https://doi.org/10.7202/1071075ar

Aller au sommaire du numéro

Éditeur(s)

Université du Québec à Montréal

ISSN

0712-8657 (imprimé)

1923-2705 (numérique)

Découvrir la revue

Citer cet article

De Serres, G., Duval, B., Shadmani, R. \& Rouleau, I. (2005). Habitudes de voyage des Québécois et des Ontariens dans les pays où l'hépatite A est endémique.

Téoros, 24(3), 12-18. https://doi.org/10.7202/1071075ar 


\section{Habitudes de voyage des Québécois et des Ontariens dans les pays où I'hépatite $A$ est endémique}

\section{Gaston De Serres, Bernard Duval, Ramak Shadmani et Isabelle Rouleau}

Environ 20 millions de voyages à l'extérieur du Canada se font chaque année. Bien que cette tendance soit à la hausse en 2005, le nombre de voyages vers les États-unis, quant à lui, diminue (Gagnon, 2005). Conséquemment, le nombre de voyages vers des pays tropicaux ou sous-tropicaux, où les conditions hygiéniques s'avèrent parfois différentes de celles du Canada, sont en hausse.

Les risques de contracter une maladie infectieuse lors d'un voyage à l'étranger dépendent de bon nombre de facteurs, tels que l'âge et le sexe du voyageur, son état de santé, son itinéraire, la durée et le genre de voyage ${ }^{1}$. Certaines de ces maladies (l'hépatite $A[H A]$ est la plus fréquente) peuvent toutefois être évitées grâce à la vaccination (Wolfe, 1995 ; Steffen, 1992). Pourtant, malgré les campagnes de sensibilisation et la disponibilité des vaccins contre cette maladie, seulement $15 \%$ des voyageurs se font vacciner avant de quitter le pays (De Serres et al., 2002).

On estime qu'environ 1845 cas d'HA sont diagnostiqués au Canada chaque année et que près du tiers de ces infections auraient été contractées lors d'un voyage à l'étranger (Teitelbaum, 2004). L'HA est une maladie du foie causée par un virus qui se transmet au contact d'eau ou d'aliments contaminés par des excréments humains ou encore au contact de personnes infectées par le virus ; environ $70 \%$ des personnes infectées par le virus de l'HA présenteront des symptômes qui apparaîtront, en moyenne,

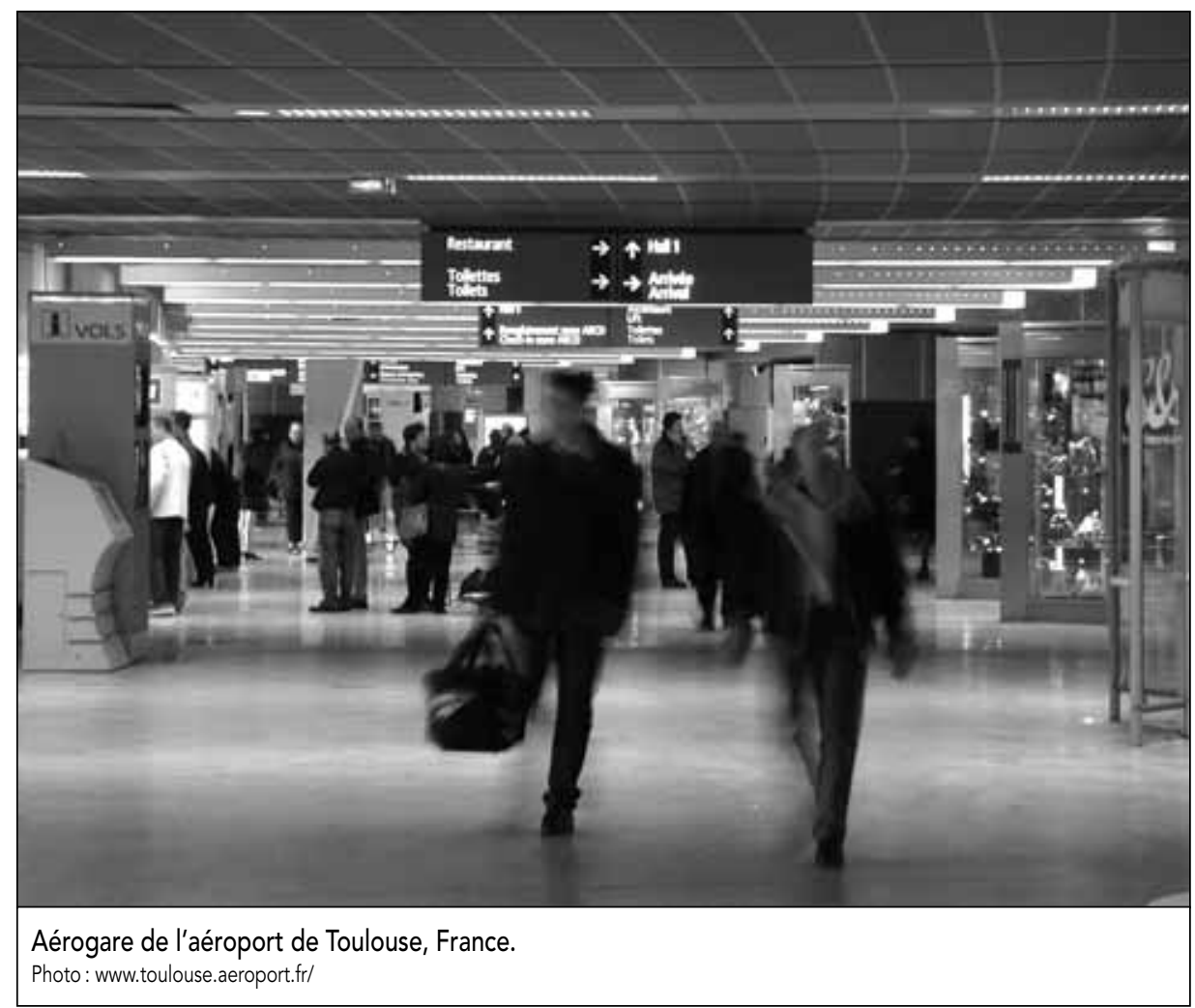

dans les trente jours suivant l'infection (Heymann, 2004). Les personnes infectées peuvent souffrir de fièvre, de douleurs abdominales, de nausées et de vomissements et même développer une jaunisse. Les symptômes, parfois sévères, peuvent durer jusqu'à deux mois et les personnes atteintes peuvent transmettre la maladie à leurs proches.

L'HA est hautement endémique, c'est-àdire que la maladie se transmet de façon continue et importante dans tous les pays d'Amérique latine et des Caraïbes, d'Afrique et d'Asie (sauf le Japon) et dans la majorité des pays de l'Europe de l'Est. Le Mexique, la République dominicaine et
Cuba ne constituent donc que quelquesuns des pays où l'HA est endémique (De Serres et al., 2002). Les voyageurs les plus à risque de contracter la maladie sont ceux qui voyagent longtemps et dans des conditions hygiéniques sous-optimales (Steffen et al., 1994). Toutefois, puisque les voyageurs les plus à risque ne représentent qu'une faible fraction de l'ensemble des voyageurs, la majorité des cas d'HA contractés à l'étranger et diagnostiqués au Canada surviennent chez les voyageurs qui ont effectué un voyage de moins de deux semaines dans des conditions jugées à faible risque (ex. : centre de villégiature tout inclus, voyage de groupe organisé, etc.) (De Serres et al., 2002). 
Afin de mieux orienter les campagnes de sensibilisation et de prévention de l'HA auprès des voyageurs canadiens qui sont à risque de contracter cette maladie, il est impératif que des données de qualité sur leurs habitudes de voyage soient accessibles. Les objectifs de notre projet de recherche consistaient à décrire les habitudes de voyage des citoyens de l'Ontario et du Québec qui ont effectué un voyage au cours des années 1990 à 1999, dans un pays où l'HA est considérée comme endémique.

\section{Méthodologie}

\section{Participants}

L'étude a été réalisée auprès d'adultes résidant au Québec ou en Ontario, qui ont été sélectionnés par la méthode de composition téléphonique aléatoire. Dans chaque ménage, on interrogeait un seul adulte (18 ans +). S'il y avait plus d'un adulte, on sélectionnait le répondant de façon aléatoire à l'aide d'un programme informatique. Un total de 12067 numéros de téléphone ont été générés. De ce nombre, 3228 n'étaient pas en service lors du recrutement, 1357 se sont avérés être des numéros commerciaux, 986 sont demeurés sans réponse malgré six à dix appels. Puisque seuls les ménages où un adulte ( $\geq 18$ ans) pouvait s'exprimer en français ou en anglais étaient admissibles, 533 ménages supplémentaires se sont révélés inadmissibles. Afin d'assurer la représentativité des résultats obtenus, l'échantillon a été pondéré en fonction du poids relatif de chaque province et des principales régions métropolitaines (Toronto et Montréal).

Des 5863 ménages (3658 ménages ontariens et 2205 ménages québécois) où il y avait une personne admissible, 4002 (68\% des personnes interrogées) individus ont accepté de participer à l'étude.

\section{Données recueillies}

Les renseignements ont été recueillis sur l'ensemble des voyages effectués de 1990 à 1999 dans les pays où l'HA était endémique. Les répondants devaient identifier le nombre de voyages qu'ils avaient faits dans ces pays, la destination ainsi que la date et la durée du voyage. On se limitait à un maximum de dix voyages durant la pé- riode à l'étude. Les participants devaient fournir les renseignements suivants : âge, sexe, niveau de scolarité, tranche de revenus et pays de naissance (pour ceux nés hors du Canada, on demandait l'année d'entrée au pays). Par ailleurs, pour les trois voyages les plus récents, on recueillait aussi :

- les motifs de voyage (tourisme, travail ou travail de bénévole) ;

- le niveau d'organisation du voyage (forfait tout organisé, partiellement organisé, auto-organisé) ;

- le type d'hébergement et la durée de séjour dans chaque type d'hébergement ;

- le type d'établissement de restauration et le nombre de repas consommés dans chaque type d'établissement ;

- la conscience des risques de maladies évitables par la vaccination.

\section{Analyses statistiques}

Chaque voyage effectué a été classé dans l'une ou l'autre des catégories de risque suivantes:

- voyage chez des amis et des membres de la famille (AMF) (voyage où plus de $50 \%$ des nuitées ont eu lieu au domicile d'amis ou de membres de la famille);
- voyage à faible risque (voyage de 2 semaines ou moins passées dans un établissement de première classe) ;

- voyage à haut risque (voyage de plus de 4 semaines, où $50 \%$ des nuitées ont eu lieu dans un établissement à faible budget) ;

- voyage à risque intermédiaire (voyage n'appartenant à aucune autre catégorie de risque).

La proportion des repas pris dans chacun des types d'établissement étant fortement corrélée avec le type d'hébergement choisi, seul le type d'hébergement a été inclus dans les analyses, puisqu'on estime que celui-ci est moins sujet au biais de rappel. Les proportions ont été comparées à l'aide de tests du $x^{2}$ de Mantel-Haenzsel, du test exact de Fisher ou du $x^{2}$ de Pearson, selon le cas.

\section{Résultats}

\section{Caractéristiques des participants}

De janvier 1990 à novembre 1999, 1070 (24\%) répondants rapportent avoir voyagé au moins une fois dans un pays où l'HA est endémique, pour un total de 2444 voyages. De plus en plus d'adultes voyagent, puisque leur proportion passe de $3 \%$ en 1990 à $9 \%$ en 1999 (Figure 1). De 1990 à 1999, la proportion des résidants du Québec qui ont ef-

\section{Graphique 1}

Proportion des adultes qui ont voyagé dans un pays où l'HA est endémique, selon la province de résidence ou l'origine

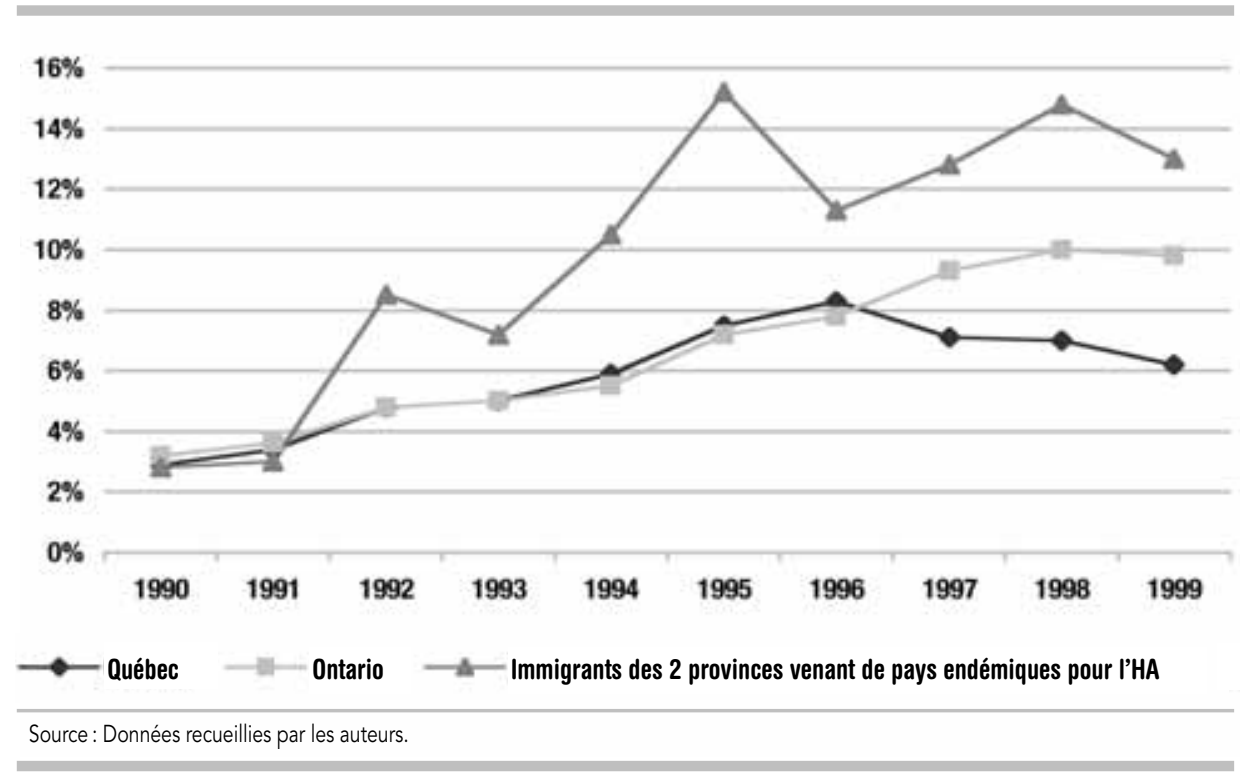


fectué un voyage (23\%) est inférieure à celle des résidants de l'Ontario (29\%), même si le nombre moyen de voyages par personne demeure similaire pour les deux provinces (2,5 voyages par personne au Québec, contre 2,3 en Ontario) jusqu'en 1997. De 1997 à 1999, on note une plus grande proportion d'Ontariens qui ont voyagé (Graphique 1). Les voyageurs interrogés

\section{Tableau 1}

Caractéristiques personnelles des répondants et du sous-groupe des voyageurs

\begin{tabular}{|c|c|c|c|}
\hline Caractéristique & $\begin{array}{c}\text { Répondants } \\
n=4002\end{array}$ & $\begin{array}{c}\% \text { Voyageurs } \\
n=1070\end{array}$ & valeur $P$ \\
\hline \multicolumn{4}{|l|}{ Sexe } \\
\hline Hommes & 1798 & $28 \%$ & \multirow{2}{*}{0,24} \\
\hline Femmes & 2204 & $26 \%$ & \\
\hline \multicolumn{4}{|l|}{ Province } \\
\hline Québec & 1673 & $23 \%$ & \multirow{2}{*}{0,0001} \\
\hline Ontario & 2329 & $29 \%$ & \\
\hline \multicolumn{4}{|l|}{ Pays d'origine } \\
\hline Canada & 3251 & $24 \%$ & \multirow{3}{*}{0,0001} \\
\hline Où l'HA est endémique & 401 & $47 \%$ & \\
\hline Où l'HA n'est pas endémique & 350 & $31 \%$ & \\
\hline \multicolumn{4}{|l|}{ Groupe d’âge } \\
\hline $18-24$ ans & 458 & $24 \%$ & \multirow{5}{*}{0,0001} \\
\hline $25-34$ ans & 719 & $34 \%$ & \\
\hline $35-44$ ans & 938 & $29 \%$ & \\
\hline $45-54$ ans & 738 & $29 \%$ & \\
\hline 55 ans et plus & 1058 & $19 \%$ & \\
\hline \multicolumn{4}{|l|}{ Niveau de scolarité (en années) } \\
\hline$<12$ années & 838 & $12 \%$ & \multirow{4}{*}{0,0001} \\
\hline 12 années & 980 & $21 \%$ & \\
\hline 13-15 années & 1113 & $28 \%$ & \\
\hline$\geq 16$ années & 994 & $45 \%$ & \\
\hline \multicolumn{4}{|l|}{ Revenu familial annuel } \\
\hline$<20000 \$$ & 730 & $12 \%$ & \multirow{5}{*}{0,0001} \\
\hline 20000 à $39999 \$$ & 905 & $21 \%$ & \\
\hline 40000 à $59999 \$$ & 735 & $31 \%$ & \\
\hline 60000 à $99999 \$$ & 540 & $41 \%$ & \\
\hline$\geq 100000 \$$ & 310 & $57 \%$ & \\
\hline \multicolumn{4}{|l|}{$\begin{array}{l}\text { Intentions de voyages futurs en } \\
\text { pays où l'HA est endémique }\end{array}$} \\
\hline Dans la prochaine année (2000) & 827 & $60 \%$ & 0,0001 \\
\hline Dans les 5 prochaines années (2000-2004) & 1674 & $51 \%$ & 0,0001 \\
\hline
\end{tabular}

Source : Données recueillies par les auteurs. rapportent avoir fait un seul (48 \%), 2 (22\%) ou 3 voyages (14\%) au cours de la période visée, alors que $16 \%$ disent avoir effectué 4 voyages ou plus au cours de la même période. Les répondants qui ont le plus voyagé au cours de la période étaient âgés de 25 à 34 ans, avaient un niveau de scolarité supérieur à 16 années et un revenu familial annuel supérieur (tableau 1).

\section{des pays où l'hépatite $A(H A)$ est endémique
daracteristiques personn}

\section{Caractéristiques des voyages effectués, selon la destination}

Les résultats de l'étude montrent que la majorité des voyages sont touristiques (86\%) et ont plus souvent lieu en Amérique latine et dans les Caraïbes (90\% des séjours touristiques) (tableau 2). Les pays les plus souvent visités sont le Mexique $(24 \%$ de tous les voyages effectués), la République dominicaine (10\%), Cuba (8\%) et la Jamaïque (5\%). II y a 3 fois plus d'Ontariens que de Québécois qui choisissent la Jamaïque comme destination voyage (7\% contre $2 \%$ ), alors que 3 fois plus de Québécois choisissent la République dominicaine (16\% contre $6 \%$ ).

Par ailleurs, les voyages tout organisés sont plus fréquents en Amérique latine et dans les Caraïbes (50\%) que dans les autres régions et représentent près de $59 \%$ des voyages effectués au Mexique, en République dominicaine, à Cuba et en Jamaïque. À l'inverse, les voyages auto-organisés sont plus fréquents parmi les séjours en Afrique (61\%), en Asie (71\%) ou en Europe de l'Est (68\%) (tableau 2).

Les voyages où toutes les nuitées sont passées dans un établissement de première classe sont plus fréquents en Amérique (49\%) que dans les autres destinations (17\% à $20 \%)$. Les voyages où plus de $50 \%$ des nuitées sont chez des amis et des membres de la famille sont d'environ $10 \%$ pour les voyages en Amérique, $52 \%$ en Europe de l'Est, $41 \%$ en Afrique et $46 \%$ en Asie. Les voyages où plus de $50 \%$ des nuitées ont lieu dans un établissement à faible budget sont de 3 à 4 fois moins fréquents en Amérique que sur les autres continents (3\% contre 12-17\%).

\section{Caractéristiques des voyages en fonction du motif de départ}

Les voyages d'affaires sont généralement d'une durée supérieure aux voyages à visée touristique et sont plus souvent effectués par des voyageurs âgés de 35 à 44 ans dont la scolarité et le revenu sont supérieurs à ceux des autres voyageurs (tableau 3). Toutefois, ce sont les voyageurs qui vont faire du travail bénévole qui font les séjours les plus longs. Ces voyageurs sont aussi généralement plus jeunes et moins fortunés. 
Tableau 2

Caractéristiques des voyages effectués et des voyageurs en fonction des continents/pays visités

\begin{tabular}{|c|c|c|c|c|c|}
\hline Caractéristique & Amérique et les Caraïbes & Afrique & Asie & Europe de l'Est & valeur $P$ \\
\hline \multicolumn{6}{|l|}{ Sexe } \\
\hline Hommes & $838(48 \%)$ & $54(48 \%)$ & $133(56 \%)$ & $75(44 \%)$ & \multirow{2}{*}{0,11} \\
\hline Femmes & $901(52 \%)$ & $59(52 \%)$ & $106(44 \%)$ & $94(56 \%)$ & \\
\hline \multicolumn{6}{|l|}{ Pays d'origine } \\
\hline Canada & $1379(79 \%)$ & $56(50 \%)$ & $108(45 \%)$ & $107(7 \%)$ & \multirow{3}{*}{0,0001} \\
\hline Où le HA est endémique & $202(12 \%)$ & $41(36 \%)$ & $93(39 \%)$ & $51(30 \%)$ & \\
\hline Où l'HA n'est pas endémique & $158(9 \%)$ & $16(14 \%)$ & $38(16 \%)$ & $11(7 \%)$ & \\
\hline \multicolumn{6}{|l|}{ Groupe d’âge } \\
\hline $18-24$ ans & $232(14 \%)$ & $28(25 \%)$ & $37(16 \%)$ & $30(18 \%)$ & \multirow{5}{*}{0,01} \\
\hline $25-34$ ans & $491(30 \%)$ & $28(25 \%)$ & $64(27 \%)$ & $39(24 \%)$ & \\
\hline $35-44$ ans & $422(26 \%)$ & $19(17 \%)$ & $55(23 \%)$ & $49(30 \%)$ & \\
\hline $45-54$ ans & $311(19 \%)$ & $19(17 \%)$ & $44(19 \%)$ & $20(12 \%)$ & \\
\hline 55 ans et plus & $188(11 \%)$ & $17(15 \%)$ & $35(15 \%)$ & $26(16 \%)$ & \\
\hline \multicolumn{6}{|l|}{ Niveau de scolarité (en années) } \\
\hline$<12$ années & $166(10 \%)$ & $5(4 \%)$ & $17(7 \%)$ & $10(6 \%)$ & \multirow{4}{*}{0,000} \\
\hline 12 années & $333(19 \%)$ & $14(12 \%)$ & $27(11 \%)$ & $18(11 \%)$ & \\
\hline 13-15 années & $521(30 \%)$ & $27(24 \%)$ & $39(16 \%)$ & $34(20 \%)$ & \\
\hline$\geq 16$ années & $712(41 \%)$ & $67(59 \%)$ & $156(65 \%)$ & $107(63 \%)$ & \\
\hline \multicolumn{6}{|l|}{ Revenu familial annuel } \\
\hline$<20000 \$$ & $84(6 \%)$ & $12(13 \%)$ & $15(7 \%)$ & $8(6 \%)$ & \multirow{5}{*}{0,008} \\
\hline 20000 à $39999 \$$ & $268(18 \%)$ & $23(24 \%)$ & $31(15 \%)$ & $23(17 \%)$ & \\
\hline 40000 à 59999 \$ & $378(26 \%)$ & $25(26 \%)$ & $44(22 \%)$ & $27(19 \%)$ & \\
\hline 60000 à $99999 \$$ & $366(25 \%)$ & $23(24 \%)$ & $63(31 \%)$ & $51(37 \%)$ & \\
\hline$\geq 100000 \$$ & $367(25 \%)$ & $13(14 \%)$ & $51(25 \%)$ & $30(22 \%)$ & \\
\hline \multicolumn{6}{|l|}{ Motif de voyage } \\
\hline Travail & $119(9 \%)$ & $15(16 \%)$ & $42(21 \%)$ & $27(19 \%)$ & \multirow{3}{*}{0,0001} \\
\hline Tourisme & $1248(90 \%)$ & $68(74 \%)$ & $152(76 \%)$ & $106(75 \%)$ & \\
\hline Travail de bénévole & $21(2 \%)$ & $9(10 \%)$ & $6(3 \%)$ & $9(6 \%)$ & \\
\hline \multicolumn{6}{|l|}{ Type de voyage } \\
\hline Tout organisé & $698(50 \%)$ & $20(22 \%)$ & $35(18 \%)$ & $31(22 \%)$ & \multirow{3}{*}{0,0001} \\
\hline Partiellement organisé & $181(13 \%)$ & $15(17 \%)$ & $23(12 \%)$ & $14(10 \%)$ & \\
\hline Auto-organisé & $507(37 \%)$ & $55(61 \%)$ & $142(71 \%)$ & $95(68 \%)$ & \\
\hline \multicolumn{6}{|l|}{ Durée du séjour } \\
\hline$<8$ jours & $882(51 \%)$ & $9(8 \%)$ & $34(14 \%)$ & $27(16 \%)$ & \multirow{3}{*}{0,0001} \\
\hline 8 à 14 jours & $514(30 \%)$ & $24(22 \%)$ & $47(20 \%)$ & $41(24 \%)$ & \\
\hline 15 jours ou plus & $334(19 \%)$ & $77(70 \%)$ & $157(66 \%)$ & $101(60 \%)$ & \\
\hline Conscience du risque de maladie & $761(55 \%)$ & $70(76 \%)$ & $148(74 \%)$ & $66(46 \%)$ & 0,0001 \\
\hline
\end{tabular}

Source : Données recueillies par les auteurs. 
La proportion des voyages où toutes les nuitées sont passées dans un établissement de première classe est similaire durant les voyages d'affaire et de tourisme (39\% contre $42 \%$ ). Par contre, la proportion des voyages où plus de $50 \%$ des nuitées sont passées chez des amis et des membres de la famille est 2 fois plus grande lors de voyages pour travail bénévole que pour les autres types de voyages (43\% contre $22 \%$ ).
La proportion des voyages passés en entier dans un établissement de faible budget est de 4 à 6,5 fois plus élevée chez les travailleurs bénévoles (13\%) que chez les voyageurs dont le motif du départ est le tourisme (3\%) ou les affaires (2\%), respectivement. La proportion des voyages où plus de la moitié des repas sont pris dans des établissements de fai- ble budget est similaire pour les voyages touristiques et d'affaires ( $6 \%$ contre $7 \%$ ), mais est supérieure lorsqu'il s'agit d'un voyage pour travail bénévole (13\%). Dans presque la moitié de ces derniers (49\%), plus de $50 \%$ des repas sont pris chez des amis ou de la famille, contre $22 \%$ pour les autres types de voyage.

\section{Tableau 3}

Caractéristiques des voyages effectués et des voyageurs en fonction du type de voyage

\begin{tabular}{|c|c|c|c|c|c|}
\hline Caractéristique & Total & Affaires & Tourisme & Travail de bénévole & valeur $P$ \\
\hline \multicolumn{6}{|l|}{ Sexe } \\
\hline Hommes & $936(48 \%)$ & $156(70 \%)$ & $751(45 \%)$ & $29(62 \%)$ & \multirow{2}{*}{0,0001} \\
\hline Femmes & $998(52 \%)$ & $66(30 \%)$ & $914(55 \%)$ & $18(38 \%)$ & \\
\hline \multicolumn{6}{|l|}{ Province } \\
\hline Québec & $714(37 \%)$ & $77(35 \%)$ & $614(37 \%)$ & $23(49 \%)$ & \multirow{2}{*}{0,2} \\
\hline Ontario & $1220(52 \%)$ & $145(65 \%)$ & $1051(63 \%)$ & $24(51 \%)$ & \\
\hline \multicolumn{6}{|l|}{ Pays d'origine } \\
\hline Canada & $1406(73 \%)$ & $157(71 \%)$ & $1208(73 \%)$ & $41(87 \%)$ & \multirow{3}{*}{0,0004} \\
\hline Où le HA est endémique & $331(17 \%)$ & $27(12 \%)$ & $300(18 \%)$ & $4(9 \%)$ & \\
\hline Où le HA n'est pas endémique & $197(10 \%)$ & $38(17 \%)$ & $157(9 \%)$ & $2(4 \%)$ & \\
\hline \multicolumn{6}{|l|}{ Niveau de scolarité (en années) } \\
\hline$<12$ années & $169(9 \%)$ & $6(3 \%)$ & $157(9 \%)$ & $6(13 \%)$ & \multirow{4}{*}{0,0001} \\
\hline 12 années & $350(18 \%)$ & $20(9 \%)$ & $326(20 \%)$ & $4(19 \%)$ & \\
\hline 13-15 années & $548(28 \%)$ & $57(26 \%)$ & $476(29 \%)$ & $15(32 \%)$ & \\
\hline$\geq 16$ années & $858(45 \%)$ & $137(62 \%)$ & $699(42 \%)$ & $22(47 \%)$ & \\
\hline \multicolumn{6}{|l|}{ Revenu familial annuel } \\
\hline$<20000 \$$ & $126(8 \%)$ & $4(2 \%)$ & $117(8 \%)$ & $5(13 \%)$ & \multirow{5}{*}{0,0001} \\
\hline 20000 à $39999 \$$ & $311(19 \%)$ & $29(16 \%)$ & $266(19 \%)$ & $16(41 \%)$ & \\
\hline 40000 à $59999 \$$ & $401(24 \%)$ & $44(25 \%)$ & $352(25 \%)$ & $5(13 \%)$ & \\
\hline 60000 à $99999 \$$ & $433(26 \%)$ & $44(25 \%)$ & $378(27 \%)$ & $11(28 \%)$ & \\
\hline$\geq 100000 \$$ & $368(22 \%)$ & $58(32 \%)$ & $308(22 \%)$ & $2(5 \%)$ & \\
\hline \multicolumn{6}{|l|}{ Type de voyage } \\
\hline Tout organisé & $816(43 \%)$ & $85(39 \%)$ & $736(44 \%)$ & $5(11 \%)$ & \multirow{3}{*}{0,0001} \\
\hline Partiellement organisé & $526(27 \%)$ & $42(19 \%)$ & $468(28 \%)$ & $16(34 \%)$ & \\
\hline Auto-organisé & $573(30 \%)$ & $93(42 \%)$ & $454(27 \%)$ & $26(55 \%)$ & \\
\hline \multicolumn{6}{|l|}{ Durée du séjour } \\
\hline$<8$ jours & $826(43 \%)$ & $85(39 \%)$ & $736(44 \%)$ & $5(11 \%)$ & \multirow{3}{*}{0,0001} \\
\hline 8 à 14 jours & $526(27 \%)$ & $42(19 \%)$ & $468(28 \%)$ & $16(34 \%)$ & \\
\hline 15 jours ou plus & $573(30 \%)$ & $92(42 \%)$ & $454(27 \%)$ & $26(55 \%)$ & \\
\hline Conscience du risque de maladie & $1105(58 \%)$ & $144(66 \%)$ & $926(56 \%)$ & $35(74 \%)$ & 0,0001 \\
\hline
\end{tabular}




\section{Conscience des risques pour la santé}

Les trois quarts des individus qui ont voyagé en Afrique et en Asie étaient conscients des risques pour la santé associés aux voyages. Toutefois, moins de la moitié des individus qui ont voyagé en Europe de l'Est étaient conscients de ces risques (tableau 2).

La proportion de voyageurs conscients des risques pour la santé encourus lors de voyages à l'étranger est supérieure chez les individus qui effectuent des voyages pour travail bénévole (74\%) que chez les voyageurs d'affaires (66 \%) et les touristes (56 \%) (tableau 3).

\section{Intention de voyager dans un pays où l'HA est endémique}

L'intention de voyager dans des pays où l'HA est endémique varie selon les régions, mais est généralement plus élevée dans les régions métropolitaines comme Toronto et Montréal. De l'ensemble des répondants ( $n=4002), 21 \%$ des participants ont l'intention de voyager dans un pays où l'HA est endémique durant la prochaine année (2000) et $44 \%$ ont l'intention d'y aller d'ici les 5 prochaines années (2000-2004). Chez les voyageurs par contre ( $n=1070)$, l'intention de voyager à nouveau dans la prochaine année (46 \%) ou d'ici les 5 prochaines années (76\%) est supérieure.

\section{Discussion}

À notre connaissance, peu d'études de population se sont intéressées aux habitudes de voyage des Canadiens au cours des dix dernières années. Notre étude permet de décrire les habitudes de voyage des résidants de l'Ontario et du Québec. Puisque les répondants ont été sélectionnés par méthode de composition aléatoire et que l'échantillon a été pondéré en fonction du poids relatif de chaque province et de leur principale région métropolitaine, il est raisonnable de croire que notre étude donne un portrait juste des habitudes de voyage de ces deux provinces.

Nos résultats permettent de démontrer que, de 1990 à 1999, environ un quart des résidants du Québec et de l'Ontario ont visité un pays où l'HA était endémique. Bien que les deux provinces présentent la même proportion de voyageurs, il semble que les résidants de l'Ontario qui font des voyages en font plus que les Québécois et que les choix de destinations varient aussi entre les deux provinces.
De plus en plus de voyageurs séjournent chez des amis et des membres de la famille. En effet, près de la moitié des voyageurs qui se rendent outre-mer ainsi que les gens qui voyagent pour faire du travail bénévole séjournent plus souvent chez des amis et des membres de la famille que ceux qui voyagent pour d'autres motifs. Ainsi, près de 50 millions d'individus provenant de pays industrialisés visitent annuellement des pays en développement (Ryan et Kain, 2000) et cette tendance est en croissance (Kollaritsch et Widermann, 1992). De 1996 à 1999, au Québec et en Ontario, de $6 \%$ à $10 \%$ des adultes chaque année ont voyagé dans des pays où l'HA était endémique. Afin de diminuer les risques de contracter une infection lors d'un séjour à l'étranger et d'obtenir les conseils, les traitements préventifs et la couverture vaccinale appropriée, on recommande aux voyageurs de rencontrer un professionnel de la santé (Reed et al., 1994). La médecine du voyage étant en constant changement, les médecins qui se spécialisent dans ce domaine sont souvent les mieux outillés pour conseiller les voyageurs sur les précautions à prendre avant un séjour hors du pays (Dos Santos et al., 1999). Toutefois, seulement $15 \%$ des voyages sont précédés d'une visite dans une clinique de médecine du voyage (Duval et al., 2003).

\section{Conclusion}

Avec l'augmentation rapide de l'immigration en provenance de pays en développement et la diminution des coûts de transport, on peut s'attendre à une augmentation du nombre de Canadiens qui voyagent à l'étranger et séjournent chez des amis et des membres de la famille. Comparativement aux autres types de voyageurs, ceux-ci sont non seulement plus susceptibles de faire des voyages de longue durée et de consommer de la nourriture et des boissons contaminées, mais ils sont aussi moins susceptibles de rencontrer un spécialiste en médecine du voyage pour obtenir les services de santé appropriés (Fulford et Keystone, 2005).

Les travailleurs de l'industrie du voyage occupent une position de choix qui leur permet d'informer et de conscientiser les voyageurs sur les risques de santé qu'ils encourent (Kollaritsch et Widermann,1992; Dos Santos et al., 1999; Provost et Soto, 2001 ; Venne, 1994 ; Cossar et al., 1990). Les habitudes de voyage décrites ici nous permettent d'identifier les voyageurs les plus à risque et d'outiller les travailleurs de l'industrie du voyage afin de leur permettre de jouer leur rôle d'intervenantpivot entre les cliniques de médecine du voyage et le voyageur. Ainsi, l'augmentation des efforts de sensibilisation auprès de la clientèle pourrait protéger non seulement la santé de ces individus, mais aussi celle de leurs contacts, et ainsi contribuer à améliorer la santé publique (Angell et Behrens, 2005).

Gaston De Serre, Institut national de santé publique du Québec et Unité de recherche en santé publique, Centre de recherche du CHUL-CHUQ, Université Laval.

Bernard Duval, Institut national de santé publique du Québec et Unité de recherche en santé publique, Centre de recherche du CHUL-CHUQ, Université Laval.

Ramak Shadmani, Unité de recherche en santé publique, Centre de recherche du CHUL-CHUQ, Université Laval.

Isabelle Rouleau, Unité de recherche en santé publique, Centre de recherche du CHUL-CHUQ, Université Laval.

\section{Notes}

1 Information sur la maladie - Hépatite A. Agence de Santé publique du Canada, [http://www.phac-aspc.gc.ca/hcai-iamss/ bbp-pts/hepatitis/hep_a_f.html], consulté le 25 août 2005.

\section{Bibliographie}

Angell, S.Y., et R.H. Behrens (2005), "Risk Assessment and Disease Prevention in Travelers Visiting Friends and Relatives", Infectious Disease Clinics of North America, vol. 19, n० 1, p. 49-65.

Cossar, J.H., D. Reid, et R.J. Fallon (1990), «A Cumulative Review of Studies on Travelers, Their Experience of IIIness and the Implications of These Findings ", Journal of Infectious Diseases, vol. 21, p. 27-42.

De Serres, G., B. Duval, R. Shadmani, N. Boulianne, G. Phoani, M. Naus, et al. (2002), "Ineffectiveness of the Current Strategy to Prevent Hepatitis A in Travelers ", Journal of Travel Medicine, vol. 9, p. 10-16.

Dos Santos, C.C., A. Anvar, J.S. Keystone, et K.C. Kain (1999), « Survey of Use of Malaria Prevention Measures by Canadians Visiting India", Canadian Medical Association Journal, vol. 160, p. 195-200. 
Duval, B, G. De Serres, R. Shadmani, N. Boulianne, G. Pohani, M. Naus, et al

Journal of Travel Medicine, vol. 10, p. 4-10.

Fulford, M., et J.S. Keystone (2005), "Health Risks Associated With Visiting Friends and Relatives in Developing Countries ", Current Infectious Disease Report, vol. 7, p. 48-53.

Gagnon, M. (2005), «Affluence des visiteurs », Le Quotidien, 20 juillet.

Heymann, D.L. (2004), Control of Communicable Diseases Manual, $18^{\text {th }}$ Edition, Washington DC, American Public Health Association, 700 p.

Kollaritsch, H., et G. Widermann (1992), " Compliance of Austrian Tourists With Prophylactic Measures ", European Journal of Epidemiology, vol. 8, p. 243-251.
Provost, S., et J. Soto (2001), "Predictors of Pretravel Consultations in Tourists from Quebec (Canada) ", Journal of Travel Medicine, vol. 8, p. 66-75.

Reed, J.M., I.B. McIntosh, et K. Powers (1994), "Travel Illness and the Family Practitioner: A Retrospective Assessment of Travel-induced Illness in General Practice and the Effect of a Travel Illness Clinic", Journal of Travel Medicine, vol. 1, p. 192-198.

Ryan, E.T., et K.C. Kain (2000), "Health Advice and Immmunizations for Travelers ", New England Journal Medicine, vol. 342, p. 716-1725.

Steffen, R. (1992), "Risk of Hepatitis A in Travellers ", Vaccine, vol. 10, Supplément 1 , p. 69-72.
Steffen, R, M.A. Kane, C.N. Shapiro, N. Billo, K.J. Schoellhorn, et P. VanDamme (1994), "Epidemiology and Prevention of Hepatitis A in Travellers ", Journal of American Medical Association, vol. 272, p. 885-889.

Teitelbaum, P. (2004), «An Estimate of the Incidence of Hepatitis A in Unimmunized Canadian Travelers to Developing Countries ", Journal of Travel Medicine, vol. 11, p. 102-106.

Venne, S. (1994), Perception des voyageurs québécois sur leur risque de contracter certaines maladies infectieuses lors d'un séjour en République dominicaine, Montréal, Université de Montréal.

Wolfe, M.S. (1995), "Hepatitis A and the American Travelers" Journal of Infectious Diseases, vol. 171, Supplément 1, p. S29-S32.

\section{TÉQROS

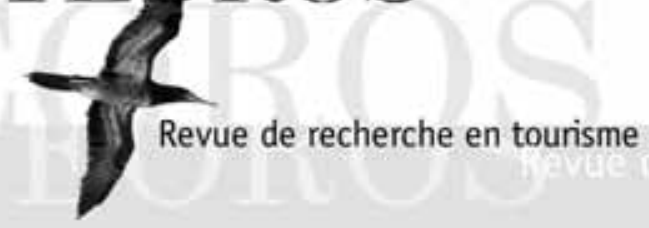

\section{Appel à textes}

TÉOROS invite tous les chercheurs et tous les professionnels qui œuvrent dans le domaine du tourisme, ou qui s'intéressent au tourisme sous tous ses aspects, à soumettre des articles de nature analytique à la revue. On peut soumettre un article en l'envoyant à :

\section{Bruno Sarrasin \\ Directeur et rédacteur en chef} TÉOROS

Département d'études urbaines et touristiques

Université du Québec à Montréal

C.P. 8888, succ. Centre-Ville

Montréal, Québec, Canada

H3С 3 P8

Courriel : teoros@uqam.ca

Tél. : (514) 987-3000, poste 6959

Publiée trois fois l'an depuis 1982, TÉOROS, revue internationale de recherche en tourisme, se veut un outil intellectuel et professionnel pour les acteurs voués au développement du tourisme. Les problématiques liées aux rapports entre le tourisme et la culture ou entre le tourisme et la société, à la gestion ou à la planification en tourisme, à des cas particuliers de mise en tourisme ainsi qu'aux questions méthodologiques d'actualité, par exemple, comptent parmi les sujets abordés. Téoros souhaite étendre le champ de ces investigations en lançant un appel élargi à ses lecteurs pour constituer, dans chacun de ses numéros, mais indépendamment de la thématique adoptée, un regroupement de tels articles de nature analytique.

Les textes soumis doivent apporter une contribution scientifique originale, que ce soit par le biais d'information factuelle jusqu'alors inconnue ou par une nouvelle interprétation d'un thème particulier. Téoros vise avant tout le transfert des connaissances; son objectif est donc de promouvoir une meilleure compréhension des phénomènes liés au tourisme auprès d'un lectorat élargi.

Les auteurs doivent faire parvenir un manuscrit présenté selon les règles de la revue, que l'on peut consulter à www.teoros.uqam.ca. Habituellement, un article analytique compte environ 4000 mots et excè- de rarement 6000 mots, avec deux illustrations; on pourra cependant considérer des textes plus longs ou plus courts.

Les articles peuvent être soumis en anglais ou en français et doivent être accompagnés d'un résumé de 100 à 200 mots, dans la langue de l'article.

Les auteurs qui n'ont pas accès au site Internet de la revue peuvent contacter la rédaction pour obtenir copie des règles de présentation qu'ils devront suivre, quant aux références, notamment. Le manuscrit doit être fourni sur support informatique (disquette ou envoi par courriel) en format RTF.

Tous les manuscrits seront évalués par un comité de lecture qui décide des articles qui seront publiés. Le comité peut faire des suggestions ou demander des modifications. La rédaction transmettra l'avis du comité aux auteurs et s'assurera que les modifications demandées seront apportées. La réponse du comité est normalement donnée dans les deux mois suivant la soumission d'un article. 